\title{
Low-frequency, one-armed oscillations in black hole accretion flows obtained from direct 3D MHD simulations
}

\author{
Mami Machida ${ }^{1}$ and Ryoji Matsumoto ${ }^{2}$ \\ ${ }^{1}$ Division of Theoretical Astronomy, National Astronomical Observatory of Japan, 2-21-1, \\ Osawa, Mitaka, Tokyo 181-8588, Japan \\ ${ }^{2}$ Department of Physics, Faculty of Science, Chiba University 1-33, Yayoi-cho, Inage-ku, \\ Chiba, 263-8522 Japan \\ email: mami@th.nao.ac.jp, matumoto@astro.s.chiba-u.ac.jp
}

\begin{abstract}
We present the results of global 3D MHD simulations of optically thin black hole accretion flows. The initial disk is embedded in a low density, spherical, isothermal halo and threaded by weak $\left(\beta \equiv P_{\text {gas }} / P_{\text {mag }}=100\right)$ toroidal magnetic field. General relativistic effects are simulated by using the pseudo-Newtonian potential. When the Maxwell stress in the innermost region of the disk is reduced due to the loss of magnetic flux or by decrease of disk temperature, inner torus is created around $4-10 r_{\mathrm{s}}$. We found that in such an inner torus, one-armed $(m=1)$ density enhancement grows and that the inner torus oscillates quasi-periodically. The oscillation period is about $0.1 \mathrm{~s}$ when we assume a $10 M_{\odot}$ black hole. This frequency agrees with the lowfrequency QPOs observed in low/hard state of black hole candidates. The disk ejects winds whose opening angle is about 30 degree. The maximum velocity of the wind is about $0.05 \mathrm{c}$.
\end{abstract}

Keywords. accretion, accretion disks - MHD - QPO

\section{Introduction}

Low/hard state in black hole X-ray binaries is characterized by the large amplitude rapid variability of X-ray flux, power law X-ray spectrum extending to hard X-rays, and the radio excess indicating the existence of outflows. In the low/hard state, lowfrequency QPOs are sometimes observed (e.g., Belloni et al. 2006). In this paper, we present the results of three-dimensional magneto-hydrodynamic (MHD) simulations of radiatively inefficient, optically thin accretion flows. Such simulations reproduced rapid time variability, low-frequency QPOs and outflows.

\section{Numerical Methods and Results}

We numerically solved resistive MHD equations by using a 3D resistive MHD code in cylindrical coordinates $(\varpi, \phi, z)$ assuming the anomalous resistivity. General relativistic effects are simulated by using the pseudo-Newtonian potential. The initial state is a disk with angular momentum $L \propto \varpi^{0.43}$ threaded by weak $\left(\beta=P_{\text {gas }} / P_{\text {mag }}=100\right)$ toroidal magnetic field. The initial disk is embedded in a low density, spherical, isothermal halo. The unit of length is the Schwarzschild radius $r_{\mathrm{s}}$, and the unit time is $t_{0}=r_{\mathrm{s}} / c=10^{-4} \mathrm{sec}$, when we assume a $10 M_{\odot}$ black hole.

Left top panel of figure 1 shows the time evolution of mass accretion rate at $\varpi=2.5 r_{\mathrm{s}}$ computed by integrating in $|z|<8 r_{\mathrm{s}}$. The mass accretion takes place due to the efficient angular momentum transport by Maxwell stress generated by the magneto-rotational instability. The mass accretion rate shows large amplitude time variations similar to the 


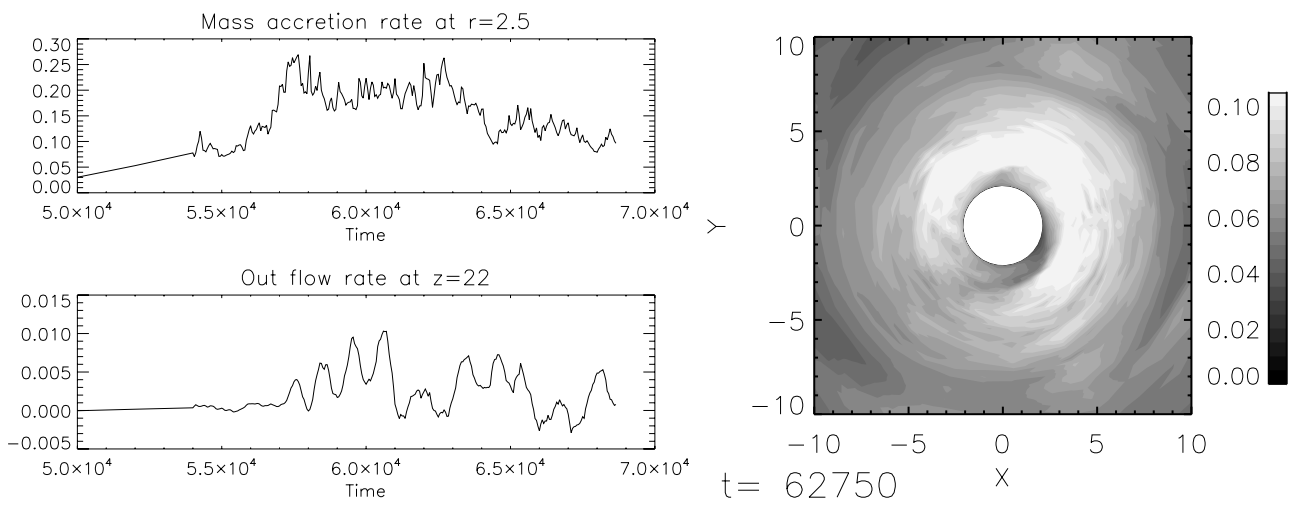

Figure 1. Left panels: The time evolution of mass accretion rate and outflow rate. top) Mass accretion rate is calculated at $\varpi=2.5$. bottom) Outflow rate measured at $z=22$. Right panel: Density distribution in $\varpi-\phi$ plane averaged in $|z|<1$.

X-ray time variations in low/hard states of black hole candidates. Left bottom panel shows the time variation of the mass outflow rate measured at $z=22 r_{\mathrm{s}}$. The mass outflow rate oscillates quasi-periodically with period $\sim 1000 t_{0}$ and correlates with the mass accretion rate with time lag around $3000 t_{0}$. The maximum speed of the outflow is $0.05 c$. Numerical results indicate that the outflow emerging from the inner torus is created intermittently by magnetic reconnections taking place in the torus. The driving mechanism of the outflow is similar to that in proto-stellar flares (Hayashi et al. 1996). In addition to the intermittent outflow, quasi-steady funnel wall wind appears around $\varpi=2.5 r_{\mathrm{s}}$. The funnel wall wind is driven by gas pressure gradient in the plunging region.

Right panel of figure 1 shows the vertically averaged density distribution of the disk. The inner torus is deformed into a crescent shape with azimuthal mode number $m=1$.

\section{Discussion}

Global 3D MHD simulations revealed that in optically thin black hole accretion flows, crescent shaped, non-axisymmetric density distribution develops in the inner torus created around $\varpi \sim 8 r_{\mathrm{s}}$. Magnetic energy release generates outflows emerging from the inner torus. The mass outflow rate shows quasi-periodic oscillation with period $0.1 \mathrm{~s}$ when the mass of the black hole is $10 M_{\odot}$. This period is comparable to the period of low-frequency QPOs observed in low/hard states.

Let us discuss whether we can observe the time variation of magnetic fields in Galactic center black hole Sgr A* . Recently, polarization of Sgr A* has been measured by sub-mm observation. These observations show the time evolution of the polarization angle. By using the formula for synchrotron self-absorption, we computed the photosphere where the optical depth $\tau=1$ at $43 \mathrm{GHz}$ and $690 \mathrm{GHz}$. Numerical results indicate that the direction of azimuthal magnetic field at $690 \mathrm{GHz}$ oscillates with period about $1000 t_{0}$. Lower frequency observation at $43 \mathrm{GHz}$ can reveal the structure of the outflowing region.

\section{References}

Belloni, T., Parolin, I. et al. 2006, MNRAS, 367, 1113

Hayashi, M., R., Shibata, K. \& Matsumoto, R. 1996, ApJ, 468, L37 